\title{
Feasibility of automated visual field examination in children between 5 and 8 years of age
}

Avinoam B Safran, Gian Luca Laffi, André Bullinger, Paolo Viviani, Catherine de Weisse, Dominique Désangles, Chantal Tschopp, Christophe Mermoud

\begin{abstract}
Aims-To investigate how young children develop the ability to undergo a visual field evaluation using regular automated perimetry.

Methods-The study included 42 normal girls aged 5, 6, 7, and 8 years. Twelve locations in the 15 degrees eccentricity were tested in one eye, using an Octopus 2000R perimeter with a two level strategy. False positive and false negative catch trials were presented. The examination was performed three times in succession. Before the examination procedure, a specially designed programme was conducted for progressive familiarisation.
\end{abstract}

Results-During the familiarisation procedure, it was found that all of the 5-yearold children, seven of the 6-year-old children, and three of the 7-year-old children were unable to perform immediately, and correctly, the instructions given during the familiarisation phase; these children took from 30 seconds to 3 minutes to comply with the examiner's requests. With the exception of one 5-year-old child, all tested subjects completed the planned procedure. The mean proportion of false negative answers in catch trials was $1.6 \%$. The mean proportion of false positive answers was $12.2 \%$. The quadratic dependency on age suggested by the averages was not significant $(F(3,116)=0.88$; $\mathbf{p}=\mathbf{0 . 4 5 )}$. Detection stimulus improved with age, as shown by the fact that probability of perceiving dim stimulus increases significantly $(F(3,116)=12.68$; p < 0.0001).

Conclusion-Children did remarkably well regarding both the duration of the examination and the reliability of the answers. A preliminary familiarisation phase with a specially designed adaptation programme was found to be mandatory with children aged 7 or under. To our knowledge, this is the first time that such an investigation has been performed.

(Br F Ophthalmol 1996;80:515-518)

Visual field examination, along with the measurement of acuity, is one of the two most important techniques used in clinical practice for evaluating visual function. In recent years, computer assisted techniques have been recognised as essential for visual field examination, in both research and patient care. Such techniques are sensitive and reliable, permit quantification of results, and allow the possibility of computer assisted data analysis.

With computerised static perimetry, extensive normative studies have been conducted in adults. ${ }^{1}$ In children, however, available reference data are scarce. In 1991, children 7 years of age or older were investigated by Bowering $e t$ $a l .{ }^{2}$ In addition, 3 years later, children aged 4 to 10 years were examined by Mutlukan and Damato, ${ }^{3}$ using both the Dicon autoperimeter and the CAMEC, a moving eye campimeter. Furthermore, in spite of controversial opinions regarding the young child's capacity for undergoing such an examination, ${ }^{4}$ no detailed investigation has been performed of the specific psychomotor developmental problems which arise in young children in such conditions.

We therefore studied the ability of young children to undergo visual field evaluation using routine automated static perimetry, and analysed various practical problems which occurred during the examination procedure.

Materials, subjects, and methods

Our investigation involved 42 girls aged 5, 6, 7, and 8 years. All subjects showed 20/20 visual acuity, and an unremarkable ophthalmic history.

Children were categorised in the following age groups: 5 years (SD 2 months), 6 years (2 months), 7 years ( 2 months), and 8 years (2 months). Ten to 12 individuals were included in each age group. Using a computer connected to the control unit of an Octopus 2000R perimeter, we developed an examination programme which included a total of 12 tested points located in the central $15^{\circ}$, on the $45-225^{\circ}$ and $135-315^{\circ}$ meridians and with $5^{\circ}$, $10^{\circ}$, and $15^{\circ}$ eccentricity.

False positive and false negative catch trials were used to evaluate subjects' attentiveness and reliability. In keeping with psychophysical testing standards, subjects were given a sound clue (click) before each test target was presented. This cue ensures increased attentiveness, but may also contribute to inappropriate responses. To assess the patient's reliability, false positive and false negative catch trials were included. To test for false positive responses, the sound cue was given with a test stimulus of subthreshold intensity. A response indicated that the patient was responding to the sound rather than to the visual stimulus, and the response was considered false positive. False negative catch trials involved presentation of maximal intensity targets at randomly 

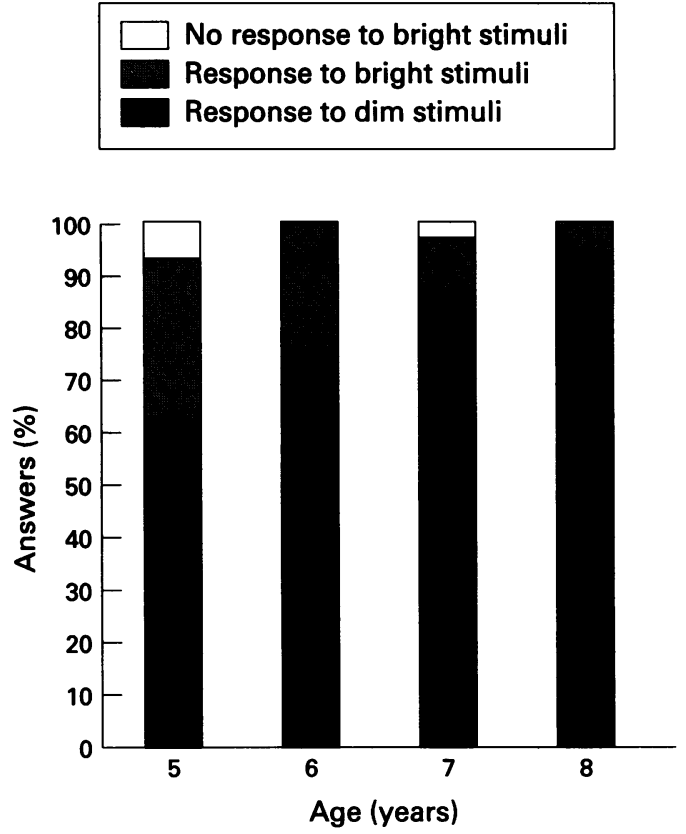

Figure 1 Respective proportions of correct and false answers to light stimuli, according to age.

selected previously determined visual field locations. Failure to respond was considered as a false negative response, indicating poor attentiveness.

In each subject, one eye was randomly selected for examination. The test procedure was as follows.

Before starting the examination, a stage of explanation and familiarisation with the testing procedure was conducted. It consisted of the successive demonstration of (1) light stimuli of different intensities, projected onto the $30^{\circ}$ eccentricity of the visual field, while the subject was attempting to gaze at the centre of the visual field while also paying attention to the lights projected onto the peripheral field; (2) the same as the preceding phase, plus the inclusion of false positive presentations; (3) the same as phase 2 , with the additional request to press the buzzer when the light stimulus was perceived; and (4) a rest period.

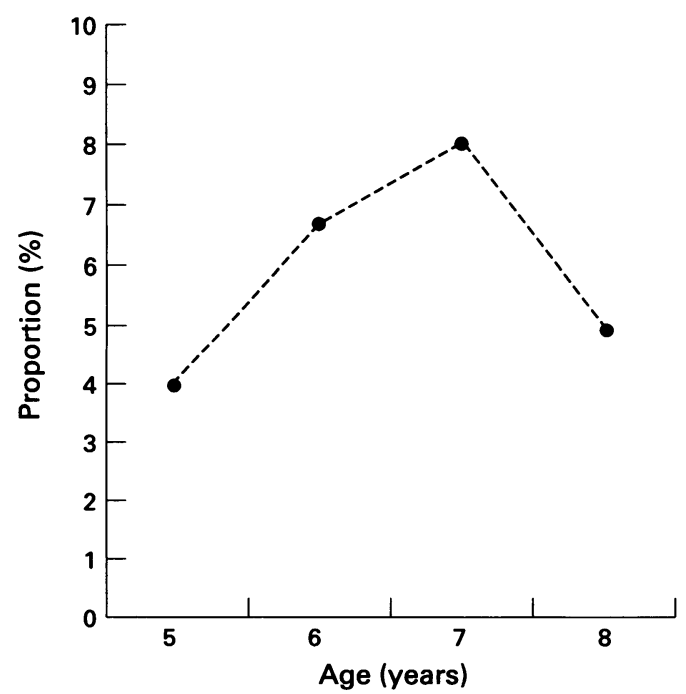

Figure 2 Proportion of false positive answers according to age.
Table 1 Number of questions presented to each individual subject according to age

\begin{tabular}{lll}
\hline Age (years) & No of subjects & $\begin{array}{l}\text { Questions (mean } \\
\text { and range) }\end{array}$ \\
\hline 5 & 9 & $140.5(109-154)$ \\
6 & 12 & $119.9(86-155)$ \\
7 & 10 & $112.6(80-144)$ \\
8 & 10 & $97.9(78-125)$ \\
\hline
\end{tabular}

Next, the examination was performed using a screening strategy consisting of a two level suprathreshold test algorithm. Firstly, we presented stimuli that were $6 \mathrm{~dB}$ brighter than those corresponding to normal mean sensitivity values $-2.5 \mathrm{SD}$, as determined for a population of 20-year-old subjects (personal data). If they were seen, sensitivity of the tested location was considered as normal. If they were not seen, stimuli were presented at a maximum intensity (1000 asb). If they were seen at this level, tested locations were considered as showing relative defects. If the stimuli were missed, defects were considered as absolute.

False positive catch trials-that is, stimuli at subliminal intensity, and false negative catch trials-that is, high intensity stimuli projected onto a location previously shown to be sensitive to dim light, were randomly included in the procedure. False positive presentations appeared randomly in $50 \%$ of the tests, but not more than twice in succession, whereas three false negative presentations occurred in each examination session.

This test procedure was performed three times in succession. The examination was also interrupted at the child's own demand.

\section{Results}

All tested subjects completed the examinations, with the exception of a 5-year-old girl who stopped the procedure 5 minutes after starting the test, as she wished to join other children playing in the next room.

All of the 5-year-old children, seven of the 6-year-old children, and three of the 7-year-old children were unable to perform immediately, and correctly, the instructions given during the familiarisation phase. They took from 30 seconds to 3 minutes to comply with the examiner's requests.

The average overall duration of the evaluation in children, including the explanation and familiarisation procedures, was about half an hour. The duration of the screening procedure ranged from 7 to 20 minutes at 5 years of age, to 5 to 12 minutes at 8 years of age. The mean duration decreased with age, from 13 minutes in 5-year-old children to 7 minutes in 8-yearold children.

The number of questions asked during each examination session is indicated in Table 1 . In each examination session, the average number of questions decreased progressively with age.

The results obtained in each age group (averaged over subjects and repetitions), are shown in Figures 1 and 2. Figure 1 demonstrates that visual detection improved with age, as shown by the fact that probability of 
perceiving dim stimulus increased significantly $(\mathrm{F}(3,116)=12.68 ; \mathrm{p}<<0.0001)$. However, there were significant differences among individuals $(F(36,80)=2.47 ; \mathrm{p}<0.0001)$. The proportion of unperceived light stimuli was small even in 5-year-old children. Figure 2 illustrates the proportion of false positive answers according to age. As indicated by the enlarged scale of this panel, false positive answers were quite rare at all ages. The quadratic dependency on age suggested by the averages was not significant $(F(3,116)=0.88 ; p=0.45)$. With respect to this measure, individuals showed significant differences within the same age group $(\mathrm{F}(36,80)=1.99 ; \mathrm{p}=0.005)$.

There were five false negative answers in a total of 308 false negative catch trials. Four 5-year-old children each gave one false negative answer, and an 8-year-old child gave one false negative answer.

\section{Discussion}

In children, normal values of sensitivity across the visual field have mainly been investigated using conventional, manual perimetry. ${ }^{5}$ Manual kinetic perimetry has the advantage of being simple to perform, mainly because of rapidity of the procedure and direct interaction between the examiner and the tested subject. But it does not allow precise quantification of sensitivity values at various locations in the visual field. Manual static perimetry might be valuable in this regard but it is a tiring procedure, which is biased by the constant intervention of the examiner. To obtain valid results using automated techniques, an effective interaction should be established between the computerised machine and the tested subject. In children, this problem is crucial.

The feasibility of conventional automated static perimetry in children has not yet been widely studied. Until now, only one study seems to have considered this problem. ${ }^{2}$ However, it involved only children aged 7 years or more. All were evaluated with an Octopus perimeter; the staircase procedure was used to determine the differential light threshold; and validity of the results was tested by assessing the reproducibility of the measured values. The authors indicated that normal 7-year-old children showed reliable results, with small interocular differences.

Our study included children from the age of 5. Considering the rapid changes which occur during the psychomotor development of the young child, ${ }^{6}$ subjects were divided into age groups with a range of 4 months. Also, considering the known difference in psychomotor development between boys and girls, only girls were included in our study in order to reduce intersex variations.

Surprisingly, all tested children except one did well when undergoing automated perimetry. They did well regarding the duration of the examination sessions. The same number of questions was asked during an examination session as during a regular screening procedure. ${ }^{7}$ The results indicate that children as young as 5 may be able to tolerate a short evaluation procedure designed for adults, pro- vided they are adequately prepared. This finding contrasted with the usual statement that, as 'children tire easily and are rapidly bored, it is necessary to work quickly, and to check rather the configuration of the field than details of visual field sizes'. 'We did not determine the maximal duration a child was able to sustain a perimetric examination. This was because, when defining the study protocol, we did not expect young children to undergo a lengthy evaluation procedure so easily, and therefore we did not design examination sessions that were lengthy enough to evaluate this variable.

In addition, the children did well regarding the reliability of the responses. To determine this reliability, we assessed the classic factors measured with automated perimetry - that is, false positive and false negative catch trials. False positive catch trials were numerous to avoid continuous presentation of suprathreshold stimuli, which might result in a non-selective, automatic answer pattern to the sound accompanying the stimulus presentation.

In this regard, we were surprised to note that, in all age groups, the rate of false negative answers was very low, and that of false positive answers was quite acceptable. ${ }^{9}$ This showed that children as young as 5 years of age gave quite reliable answers, even over a rather prolonged examination time.

Our results yield some additional information regarding the buzzer press response in evaluating children. Young patients' ability to use a buzzer during psychophysical testing is only briefly mentioned in the literature. ${ }^{5}$ Emphasising that field assessment using a preferential looking paradigm is easier in young children, Quinn et al state that the buzzer press response employed in standard clinical techniques requires complex motor and cognitive responses, which cannot be expected of the infants and juvenile patients in whom visual field information may be of clinical importance. In children aged 4 to 10 years, these authors performed kinetic perimetry with the Goldmann apparatus using the traditional buzzer for recording the perception of a visual target, and their results were highly correlated with those obtained using the preferential looking paradigm; nevertheless, they gave no detailed information on the age of the subjects in their study. In our study, the catch trial rates indicated an appropriate use of the buzzer in children as young as $\mathbf{5}$ years old. It therefore appears that conventional automated static perimetry, using the buzzer press response, can be used in evaluating such children.

The two level strategy used in this study involved the presentation of dim stimuli, followed by the projection of bright stimuli in locations where dim light was not perceived. As a result, the examination procedure was longer when detection of dim stimuli was poorer. We found that the total number of questions asked during the three examination sessions decreased progressively with age. This reflected an improvement in performance with age when undergoing automated perimetry.

The improvement in performance with age can be variously interpreted, as indicating that 
younger children have poorer visual sensitivity across the visual field, or poorer comprehension, or a lower attentional or sensorimotor performance.

In the absence of reliable reference values for normal sensitivity in young children tested with static automated perimetry, we arbitrarily used values determined for 20 -year-old subjects. Further studies of normal sensitivity values in young children are needed.

Before being tested, the children were given a systematic explanation of the procedure. This consisted of a step by step introduction, with a specific description of the projected light spot, and explanations about the existence of false positive catch trials and the principle of fixating the central target and preventing eccentric refixational saccades, while paying attention to what was happening in the peripheral visual field. This was found to be useful in preparing children below the age of 7 . This procedure should not be abandoned through fear of extending the examination time, as our results clearly showed this introductory phase to be beneficial to the examination procedure. We believe that testing small children with automated perimetry requires such an introductory procedure, which should, therefore, be available.

Finally, it should be noted that, in this study, aptitude for undergoing a conventional automated perimetry examination was evaluated in normal children. It is conceivable that children with visual changes might experience greater difficulty in undergoing such examinations. When altered areas of the visual field are tested, the procedure does, indeed, occasionally include a prolonged series of successive light projections in which the stimulus is not perceived. This can destabilise the subject's attentional state, a difficulty that is probably more pronounced in younger individuals. This issue needs to be assessed; the findings could have implications for the design of strategies to be used in children of different ages.

Presented in part at the meeting of the International Perimetric Society, Arlington, VA, USA, 6 July 1994.

Supported by the Swiss National Fund for Scientific Research, Grant No 32-36578.92.

Appreciation is expressed to the Service de la Santé de la Jeunesse of Geneva, and the children and the teachers of the Geneva public schools whose collaboration has made this research possible.

1 Heijl A, Lindgren G, Olsson J. Normal variability of the Heijl A, Lindgren G, Olsson J. Normal variability of the static perimetric threshold values across
field. Arch Ophthalmol 1987;105:1544-9.

2 Bowering E, Maurer D, Lewis TL, Brent HP. Detection thresholds in the periphery of normal and visually deprived children. [Abstract] IOVS Supplement 1991;32:962.

3 Mutlukan E, Damato BE. Computerised perimetry with moving and steady fixation in children. Eye 1993;7:554-61.

4 Verriest G, Van de Casteele J. Le champ visuel clinique. Acta Belg Arte Med Pharmac Milit 1972;18:35-205.

5 Quinn G, Fea MA, Minguini N. Visual fields in 4- to 10-yearold children using Goldmann and double-arc perimeters. $f$ Pediatr Ophthalmol Strabismus 1991;28:314-9.

6 Pratty BD. Perceptual and motor development in infants and Cratty BD. Perceptual and motor development in infants
children. Englewood Cliffs, NJ: Prentice-Hall, 1979.

7 Safran AB, Mermoud C. A neuro-ophthalmological global analysis program (N1) developed with the Octopus measurement unit. In: Heijl A, ed. Perimetry: update 1988/89. Amsterdam: Kugler and Ghedini, 1989: 151-5.

$8 \mathrm{Jenni}$ F, Flammer J. Experience with the reliability parameters of the Octopus automated perimeter. In: Greve EL, Heijl A, eds. Seventh international visual field symposium, Amsterdam, September 1986. Dordrecht: Martinus Nijhoff/DrW Junk, 1987: 601-3.

9 Whalen WR. Routine reliability parameters. In: Whalen WR Spaeth GL, eds. Computerized visual fields: what they are and how to use them. Thorofare, NJ: Slack, 1985: 81-8. 